\title{
Characteristic Radiological and Histological Patterns of Fibrous Dysplasia and Ossifying Fibroma of the Jaws at University of Nairobi Dental Teaching Hospital ${ }^{*}$
}

\author{
Jeremiah Moshy $^{1 \#}$, Elizabeth Dimba ${ }^{2}$, Tom Ocholla ${ }^{2}$, Mark Chindia ${ }^{2}$ \\ ${ }^{1}$ Department of Oral Surgery/Oral Pathology, College of Health Sciences, Muhimbili University, \\ Dar es Salaam, Tanzania \\ ${ }^{2}$ Department of Oral and Maxillofacial Surgery - Oral Pathology and Oral Medicine, \\ University of Nairobi, Nairobi, Kenya \\ Email: \#jeremiah.moshy@yahoo.com
}

Received August 10, 2011; revised October 27, 2011; accepted November 20, 2011

\begin{abstract}
Objective: To characterize the diagnostic features of ossifying fibroma (OF) and fibrous dysplasia (FD) of the jaw bones. Study Design: A histopathological and radiological analysis with full clinical documentation. Setting: University of Nairobi Dental Teaching Hospital (UNDH). Study Population: All archival cases diagnosed as FD and OF from 1992-2006 were retrieved from the UNDH Oral Pathology Laboratory records. New cases were included as they presented over a 6-month period from January to June 2007. Methodology: Information regarding the histological type of a fibro-osseous lesion (FOL) including the clinical features, demographic and radiographic data was documented for analysis; and comparison between pathological parameters and the final diagnosis was evaluated with the chi-square test. Results: FD lesions constituted 40 (27.2\%) cases while $107(72.8 \%)$ were OF. The age ranged from $1-72$ years (mean $=24.19 \pm$ SD 13 years). The differences in the gender distribution were not statistically significant. Radiographic analyses showed statistically significant differences between the appearances of the body $(p=0.012)$ and the margins $(p$ $=0.003)$ of FD and OF. The microscopic differences between the two lesions were not statistically significant. Conclusion: Differentiation between FD and OF is only possible after critically analyzing the clinical, radiological and histological criteria.
\end{abstract}

Keywords: Diagnostic; Ossifying-Fibroma; Fibrous Dysplasia; Jaws

\section{Introduction}

The term fibro-osseous lesion (FOL) is a generic designation of a group of jaw disorders (ranging from inflammatory to neoplastic in origin) that microscopically exhibit a connective tissue matrix and islands or trabeculae of bone [1,2]. Maxillofacial FOLs consist of lesions that differ with the exception of fibrous dysplasia (FD) to those found in the rest of the skeleton [3]. However, FD and ossifying fibroma (OF) are the most common FOLs occurring in the maxillofacial region [4]. FD and OF occurring in the jaws may share similarities in histomorphology and radiographic features despite their distinct patterns of disease progression. They consequently pose difficulties in classification and management [1,5].

Due to its risk for recurrence, OF should be completely enucleated from surrounding bone. FD is on the other hand managed conservatively because of its self-

*Competing Interest: No competing interest.

${ }^{\#}$ Corresponding author. limiting nature [6,7], except in syndromic cases which tend to require more aggressive surgical therapy [8]. A need for accurate diagnosis is of paramount importance for the proper management of these two divergent conditions. Radiographic examination is an essential step in differentiating between FD and OF. FD presents as a homogenous, ground-glass, radiodensity that has no clear demarcation with the surrounding bone. $\mathrm{OF}$ is in contrast a mixed radiodense and radiolucent lesion that is well demarcated from normal bone $[9,10]$. Histologically, FD is characterized by fibrous connective tissue containing trabeculae of bone in varying stages of maturity without any evidence of osteoblastic activity [11]. OF is a well demarcated lesion consisting of a fibroblastic stroma containing plexiform and lamellar bone in addition to acellular mineralized material [7,9].

FD lesions may occur clinically as monostotic, polyostotic or syndrome associated subtypes, a classification which may reflect the timing of mutations in the Gs $\alpha$ 
gene [12]. Molecular analyses of Gs $\alpha$ mutations in FD have also emerged as a definitive means of differentiation between $\mathrm{OF}$ and FD in challenging cases $[4,13]$. The aim of the present study was to characterize diagnostic features of the two most common FOLs of the jaw bones: $\mathrm{OF}$ and FD, as diagnosed in two referral hospitals in Kenya.

\section{Materials and Methods}

This investigation was approved by the Ethics, Research and Standards Committee of the Kenyatta National Hospital and the University of Nairobi-Approval No. 98/6/ 2005.

The study was carried out in the Divisions of Oral Pathology/Oral Medicine and Craniofacial Radiology at the University of Nairobi Dental Hospital (UNDH) and Kenyatta National Hospital (KNH). Paraffin embedded tissue blocks were retrieved from the archives of the histopathology unit. Histological slides of each case were analysed on the basis of selected pathologic parameters that consisted of three categories: gross features, shape and arrangement of calcified components, cellularity and pattern of non-calcified components.

Radiographs were analysed as to the anatomical location of the lesion in the jaws; the shape and status of the margins surrounding the lesion. Statistical analysis was done using the Statistical Programme for Social Sciences (SPSS Version 12.0 for Windows, 1997). Univariate com- parisons of the association between the histopathological and radiographic parameters versus the final diagnosis were evaluated with the chi-squared $\left(\mathrm{X}^{2}\right)$ test. Inferential statistics were considered significant when $\mathrm{p} \leq 0.05$.

\section{Results}

Demographic Analysis: Out of the 2649 surgical files reviewed during the 15 -year period and subsequent new cases seen over 6 months, 147 cases of FD and OF were recorded. Out of these, $40(27.2 \%)$ cases had been diagnosed as FD and $107(72.8 \%)$ as OF. Patients' ages ranged from 1 to 72 years with a median of 20 years. The mean age was 24.19 years ( $\mathrm{SD} \pm 13.3$ years). Females comprised the majority $(65.3 \%)$ of the patients at a ratio of 1:1.9. However, but this difference was not statistically significant $(p=0.108)$. FD was found to occur in the $1^{\text {st }}$ to $6^{\text {th }}$ decades of life with the $2^{\text {nd }}$ and $3^{\text {rd }}$ decades mostly affected. The pattern of occurrence of OF was essentially similar (Figure 1).

Distribution of Lesions According to Site: Seventy two cases had the specific sites of the lesions recorded. Among the mandibular lesions, $60(84.5 \%)$ were OF while $11(15.5 \%)$ were FD. In contrast, among the maxillary cases, $41(62.2 \%)$ were OF and 25 (37.9\%) were FD. One case of polyostotic FD affected both jaws (Table 1).

Radiological Evaluation: Thirty eight radiographic records of $\mathrm{OF}$ and FD were available for evaluation. OF

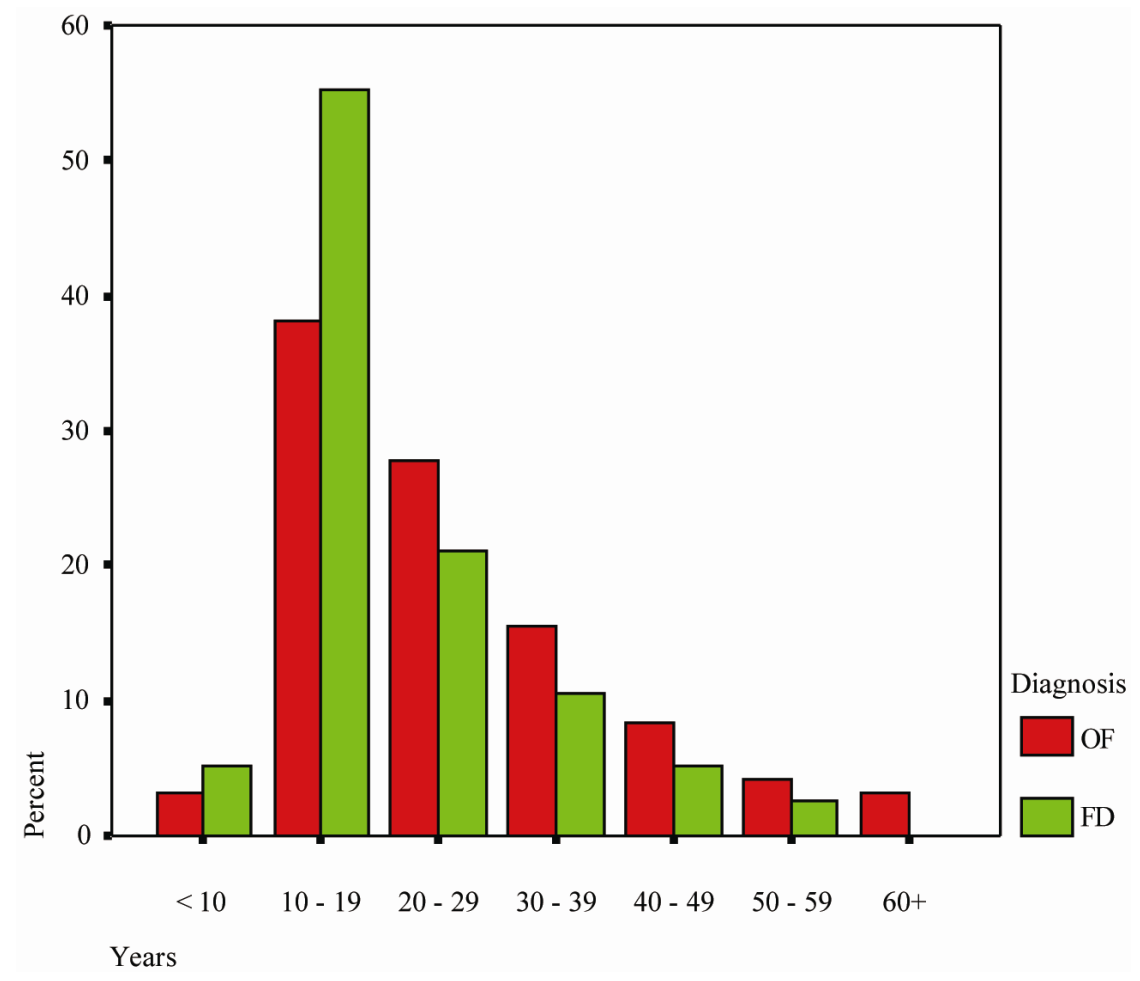

Figure 1. Distribution of the lesions according to age group. 
comprised of 26 radiographs while 12 were those of FD. OF yielded five radiographic appearances including the mixed type in 12 cases, radio-opaque 5, diffuse sclerotic 1 and ground glass appearance 4 (Figure 2). FD depicted three radiographic appearances including the mixed type 3 , the diffuse sclerotic type 4 and ground glass pattern 5 (Figure 3). The differences observed were statistically significant at $\mathrm{p}=0.012$ (Table 2). Evaluation of the extent of lesions showed that OF presented with well defined borders with sclerotic margins, well defined borders without sclerotic margins and ill-defined margins. FD presented with well defined borders without sclerotic margins and ill-defined margins. These differences were statistically significant at $p=0.003$. Common radiological features observed in both lesions were bone expansion, loss of lamina dura, and tooth displacement. Root resorption was however only observed among cases of OF.

Table 1. Distribution of lesions according to the location in the jaws.

\begin{tabular}{ccccc}
\hline Site & OF & FD & Total & Significance \\
\hline Mandible & $60(84.5 \%)$ & $11(15.5 \%)$ & $71(100 \%)$ & $\chi^{2}=8.848$ \\
Maxilla & $41(62.1 \%)$ & $25(37.9 \%)$ & $66(100 \%)$ & $\mathrm{p}=0.003^{*}$ \\
TOTAL & $\mathbf{1 0 1}(\mathbf{7 3 . 7 \% )}$ & $\mathbf{3 6 ( 2 6 . 3 \% )}$ & $\mathbf{1 3 7 ( 1 0 0 \% )}$ & \\
\hline
\end{tabular}

${ }^{*}$ Values for Chi-squared test were considered statistically significant when $\mathrm{p}$ $<0.05$.



Figure 2. Characteristic radiographic features of fibrous dysplasia of the jaws - a poorly defined mixed radiolucent/ radiopaque lesion in the left panel and "ground glass" appearance on the right panel.
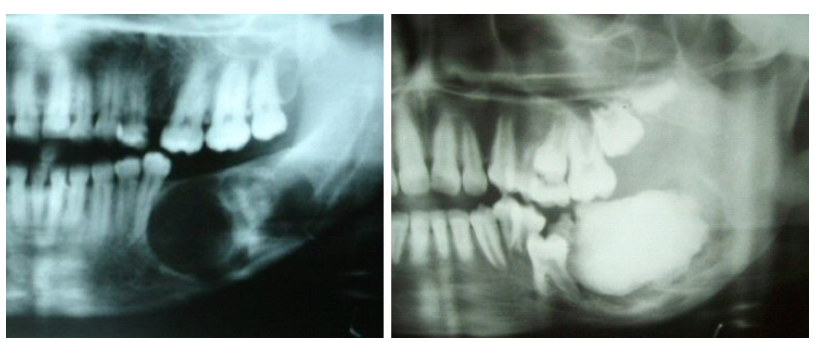

Figure 3. Characteristic radiographic features of ossifying fibroma of the jaw bones (early lesion in the left panel and late lesion in the right panel).
Histopathologic characterization: Of the gross cases of FD and OF analysed, almost all FD (93\%) cases were composed of multiple small fragments of mineralized tissue with free haemorrhage. Bone trabeculae with large osteocytes within the lacunae were present in all cases of FD while OF featured more irregular osteoid or cementoid masses (68\%) compared to $33.3 \%$ osteoid observed in FD (Figure 4). Other features found in OF included bone trabeculae with large osteocytes within the lacunae (56\%), free haemorrhage (56\%), multiple curettage fragments (48\%) and thick curvilinear trabeculae (24\%). All distinctive features were statistically significant for FD and OF (Table 3).

There were remarkable common features observed in both FD and OF including metaplastic woven bone in a fibrous stroma which was a constant feature in both lesions. Other common features for FD and OF included separate bony trabeculae, variable amounts of lamellar bone, and collagen deposits (Table 4). The study also revealed some histopathological features which were

Table 2. Radiological features characterizing OF and FD.

\begin{tabular}{lccc}
\hline \multicolumn{1}{c}{ Radiological features } & OF & FD & p Value \\
\hline \multicolumn{1}{c}{ General appearance } & & \\
Mixed type & $12(80 \%)$ & $3(20 \%)$ & \\
Radiopaque & $5(100 \%)$ & $0(0 \%)$ & $\chi^{2}=12.91$ \\
Radiolucent & $4(100 \%)$ & $0(0 \%)$ & $\mathrm{p}=0.12$ \\
Diffuse sclerotic & $1(20 \%)$ & $4(80 \%)$ & \\
Ground glass & $4(44.4 \%)$ & $5(55.6 \%)$ & \\
& & & \\
Well defined, sclerosis & $5(100 \%)$ & $0(0 \%)$ & $\chi^{2}=11.37$ \\
Well defined, no sclerosis & $18(78.3 \%)$ & $5(21.7 \%)$ & $\mathrm{p}=0.003$ \\
Poorly defined & $3(41.2 \%)$ & $4(57.1 \%)$ & \\
\multicolumn{1}{c}{ Effect on adjacent structures } & & $0.131^{\mathrm{a}}$ \\
Bone expansion & $25(67.6 \%)$ & $12(32.4 \%)$ & $0.684^{\mathrm{a}}$ \\
Loss of lamina dura & $3(75.0 \%)$ & $1(25 \%)$ & $0.625^{\mathrm{a}}$ \\
Root resorption & $5(100 \%)$ & $0(0 \%)$ & $0.289^{\mathrm{a}}$ \\
Teeth displacement & $5(55.6 \%)$ & $4(44.4 \%)$ & \\
\hline
\end{tabular}

${ }^{a}=$ Results of Fisher's exact test. Values for statistical tests were considered significant when $\mathrm{p}<0.05$.

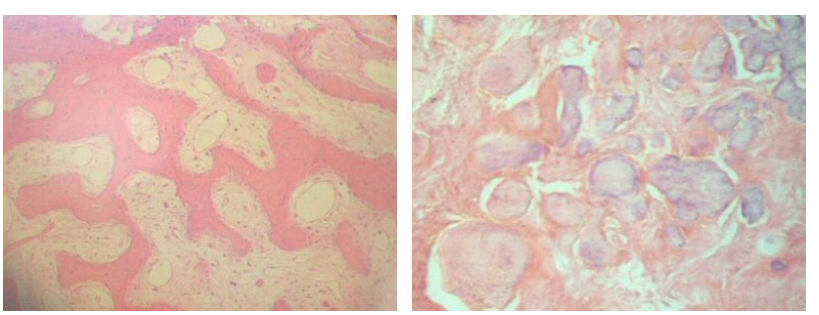

Figure 4. Histopathologic features of FD (left panel) and OF (right panel) (×400 magnification). 
Table 3. Gross and histopathological components observed in fibrous dysplasia and ossifying fibroma.

\begin{tabular}{|c|c|c|c|}
\hline Features & FD $(n=15)$ & OF $(n=25)$ & p value $^{*}$ \\
\hline \multicolumn{4}{|l|}{ Gross appearance } \\
\hline Multiple curettage fragments & $93.3 \%$ & $48.0 \%$ & 0.004 \\
\hline \multicolumn{4}{|l|}{ Calcified components } \\
\hline Thick curvilinear trabeculae & $60.0 \%$ & $24.0 \%$ & 0.023 \\
\hline Irregular mineralized masses & $33.3 \%$ & $68.0 \%$ & 0.033 \\
\hline Bone trabeculae with lacunae & $100.00 \%$ & $56.0 \%$ & 0.003 \\
\hline \multicolumn{4}{|c|}{ Non-calcified components } \\
\hline Free haemorrhage & $93.3 \%$ & $56.0 \%$ & 0.013 \\
\hline
\end{tabular}

Table 4. Similarities in histological features of OF and FD.

\begin{tabular}{|c|c|c|c|}
\hline Features & FD $(n=15)$ & OF $(n=25)$ & p value \\
\hline \multicolumn{4}{|c|}{ Gross appearance } \\
\hline Large enucleated piece(s) & $6.7 \%$ & $32.0 \%$ & 0.063 \\
\hline \multicolumn{4}{|c|}{ Calcified components } \\
\hline Separate bone trabeculae & $66.7 \%$ & $80.0 \%$ & 0.346 \\
\hline Immature bone & $100.00 \%$ & $100.0 \%$ & - \\
\hline Lamellar bone & $33.3 \%$ & $8.0 \%$ & 0.081 \\
\hline \multicolumn{4}{|c|}{ Non-calcified components } \\
\hline Storiform pattern & $33.3 \%$ & $52.0 \%$ & 0.251 \\
\hline Dense collagen & $27.6 \%$ & $24.0 \%$ & 0.851 \\
\hline Loose collagen & $73.3 \%$ & $76.0 \%$ & 0.850 \\
\hline
\end{tabular}

depicted in only FD or OF. Remarkably, $12 \%$ of the OF cases had osteoblastic rimming while none was elicited in all the FD cases. Giant cells were demonstrated in $13 \%$ of the FD cases but none were present among all the OF specimens. Further-more, 20\% of the OF lesions had a demonstrable capsule while none of the lesions in the FD group showed any evidence of encapsulation.

\section{Discussion}

The present study has shown that although some histopathological and radiographic features can separate FD from OF, it is still difficult to arrive at a definitive diagnosis by using a single diagnostic modality. There was a general predilection for FOLs to occur in the mandible. This was particularly notable for OF, which, in distributions similar to those reported by Ogunsalu et al. [14], tended to occur more frequently in the mandible, while FD tended to occur more in the maxilla. Several studies have reported different radiographic patterns for OF and FD: Ye et al. [13] reported three radiographic patterns for FD namely sclerotic, osteolytic and mixed type. Lu et al. [15] reported four radiographic patterns for OF namely cystic radiolucency, ground glass appearance, sclerotic change and mixed type while Barberi et al. [16] reported three radiographic patterns for OF namely radiolucent $(53 \%)$, sclerotic $(7 \%)$ and mixed type (40\%). Alsharif et al. [17] reported a spectrum of radiolucent, radiopaque and "cotton wool" appearances for FD and lucency, central opacification and dense opacification with sclerotic cortices for OF, which were similar to the trends observed in our study.

The pathologic distinction of FD and OF on a histopathological basis alone remains a challenge because of the extensive overlap between histopathologic features that characterized the two lesions [14]. Distinguishing FD from OF is essential since surgical treatment of the two lesions is different $[8,9]$. Cases of FD in this series were generally treated by bone recontouring, in contrast to OF which was treated by enucleation or block resection. FDs are lesions with ill-defined borders and their margins between the normal and affected parts of bone are usually poorly defined. At surgery, they tend to be removed with difficulty.

\section{Conclusion}

In this study, no single microscopic feature could render a definitive diagnosis for either FD or OF no matter how characteristic it might have seemed. Definitive diagnosis of these lesions should, therefore, be based on the critical analysis of clinical, histopathologic and radiographic findings $[9,14,17]$. Activating missense mutations of the $\alpha$ sub-unit of Gs protein found in of fibrous dysplasia are also currently utilized as diagnostic markers to distinguish it from other FOLs [18].

\section{Acknowledgements}

We are grateful to the administration of the School of Dental Sciences and Kenyatta National Hospital for permission to conduct this study.

\section{REFERENCES}

[1] F. Alawi, "Benign Fibro-Osseous Diseases of the Maxillofacial Bones. A Review and Differential Diagnosis," American Journal of Clinical Pathology, Vol. 118, 2002, pp. S50-S70.

[2] C. A. Waldron, "Fibro-Osseous Lesions of the Jaws," Journal of Oral and Maxillofacial Surgery, Vol. 51, No. 8, 1993, pp. 828-835. doi:10.1016/S0278-2391(10)80097-7

[3] D. S. Macdonald-Jankowsk, "Fibro-Osseous Lesions of the Face and Jaws," Clinical Radiology, Vol. 59, No. 1, 2004, pp. 11-25.

[4] S. Toyosawa, M. Yuki, M. Kishino, Y. Ogawa, T. Ueda, S. Murakani, E. Konishi, S. Lida, M. Kogo, T. Komor and Y. Tomita, "Ossifying Fibroma vs. Fibrous Dysplasia of the Jaw: Molecular and Immunological Characteriza- 
tion," Modern Pathology, Vol. 20, No. 3, 2007, pp. 389396. doi:10.1038/modpathol.3800753

[5] L. C. M. Keijser, T. G. van Tienen, H. W. D. Schreuder, J. A. M. Lemmens, M. J. Pruszcynski and R. P. H. Veth, "Fibrous Dysplasia of Bone: Management and Outcome of 20 Cases," Journal of Surgical Oncology, Vol. 76, No. 3, 2001, pp. 157-166. doi:10.1002/jso.1028

[6] L. Barnes, J. W. Eveson, P. Reichart and D. Sidransky, "World Health Organization Classification of Tumours Pathologyand Genetics of Head and Neck Tumours," IARC Press, Lyon, 2005.

[7] R. B. Brannon and C. B. Fowler, "Benign Fibro-Osseous Lesions: A Review of Current Concepts," Advances in Anatomic Pathology, Vol. 8, No. 3, 2001, pp. 126-143. doi:10.1097/00125480-200105000-00002

[8] P. J. Slootweg, "Lesions of the Jaws," Histopathology, Vol. 54, No. 4, 2009, pp. 401-418. doi:10.1111/j.1365-2559.2008.03097.x

[9] C.-C. Chang, H.-Y. Hung, J. Y.-F. Chang, C.-H. Yu, Y.-P. Wang, B.-Y. Liu and C.-P. Chiang, "Central Ossifying Fibroma: A Clinicopathological Study of 28 Cases," Journal of the Formosan Medical Association, Vol. 107, No. 4, 2008, pp. 288-294. doi:10.1016/S0929-6646(08)60089-3

[10] J. J. Sciubba, J. E. Fantasia and L. B. Kahn, Eds., "FibroOsseous Lesions. AFIP Atlas of Tumor Pathology. Tumors and Cysts of the Jaw," 3rd Series, Fasc 29 Edition, Armed Forces Institute of Pathology, Washington DC, 1999.

[11] M. M. Cohen Jr. and R. E. Howell, "Etiology of Fibrous Dysplasia and McCune-Albright Syndrome," International Journal of Oral and Maxillofacial Surgery, Vol. 28, No. 5, 1999, pp. 366-371. doi:10.1034/j.1399-0020.1999.285280512.x

[12] A. Sakamoto, Y. Oda, Y. Iwato and M. Tsuneyoshi, "A
Comparative Study of Fibrous Dysplasis and Osteofibrous Dysplasia with Regard to Gs $\alpha$ Mutation at the Ang201codon. Polymerase Chain Reaction-Restriction Fragment Length Polymorphism Analysis of Paraffin Embedded Tissue," Journal of Molecular Diagnostic, Vol. 2, No. 2, 2000, pp. 67-72. doi:10.1016/S1525-1578(10)60618-6

[13] X. H. Ye, "Radiologic Diagnosis and Differential Diagnosis of Fibrous Dysplasia of the Facial Bones," Chinese Journal of Radiology, Vol. 23, No. 2, 1989, pp. 86-89.

[14] C. O. Ogunsalu, A. Lewis and L. Doonquah, "Benign Fibro-Osseous Lesions of the Jaw Bones in Jamaica: Analysis of 32 Cases," Oral Diseases, Vol. 7, No. 3, 2001, pp. 155-162. doi:10.1034/j.1601-0825.2001.70304.X

[15] Y. Lu, X. Lei and Z. Zhou, "Radiologic Study of Ossifying Fibroma of the Facial and Jaw Bones," Chinese Journal of Radiology, Vol. 30, No. 2, 1995, pp. 75-77.

[16] A. Barberi, S. Cappabianca and G. Collela, "Bilateral Cemento-Ossifying Fibroma of the Maxillary Sinus," The British Journal of Radiology, Vol. 76, 2003, pp. 279-280.

[17] M. J. Alsharif, Z.-J. Sun, X.-M. Chen, S.-P. Wang and Y.-F. Zhao, "Benign Fibro-Osseous Lesions of the Jaws: A Study of 127 Chinese Patients and Review of the Literature," International Journal of Surgical Pathology, Vol. 17, No. 2, 2009, pp. 122-134. doi: $10.1177 / 1066896908318744$

[18] M. Riminucci, B. Liu, A. Corsi, A. Shenker, A. M. Speigel, P. G. Robey and P. Bianco, "The Histopathology of Fibrous Dysplasia of Bone in Patients with Activating Mutations of the Gs $\alpha$ Gene: Sit Specific Patterns and Recurrent Histological Hallmarks," Journal of Pathology, Vol. 187, No. 2, 1999, pp. 249-258. doi:10.1002/(SICI)1096-9896(199901)187:2<249::AID-P ATH222>3.0.CO;2-J 